\title{
Ordering of Ge Quantum Dots on Silicon surfaces via bottom-up and top-down approaches
}

\author{
Marco Bernardi $^{* *}$, Anna Sgarlata*, Nunzio Motta ${ }^{* *}$, Massimo Fanfoni*, Dario Del Moro* and Adalberto Balzarotti* \\ *Department of Physics, University of Roma Tor Vergata (Italy) \\ ${ }^{* *}$ Department of Built Environment and Engineering, Queensland University of Technology, Brisbane (Australia) \\ Email : marco.bernardi@qut.edu.au
}

\begin{abstract}
The nanoscale ordering of inorganic semiconductor quantum dots (QDs) is crucial to obtain reliable structures for novel nanotechnological applications such as nanomemories, nanolasers and nanoelectronic devices. We have directly grown Ge QDs by physical vapour deposition (PVD) on $\mathrm{Si}(111), \mathrm{Si}(100)$ and some of its vicinal surfaces and studied innovative bottom up techniques to order such nanostructures. Specifically, we harnessed naturally occurring instabilities due to reconstruction and intrinsic anisotropic diffusion in Si bare surfaces, such as step bunching and natural steps occurring in silicon vicinal surfaces, to order the QDs both in one dimension and in the plane. We have also shown the use of controlled quantities of surfactants, like $\mathrm{Sb}$, dramatically improves the desired ordering. Moreover, we have assisted these self-assembling processes using top-down approaches like Focused Ion Beam (FIB) milling and STM nanoindentation to control the nucleation sites and the density of the Ge QDs. Real-time study of growth and selfassembly has been accomplished using Scanning Tunneling Microscopy imaging in UHV. An explanation of the occurring processes is given, and a software routine is used to quantify the ordering of the QDs both in pre-patterned and bare surfaces. Applications, mainly in the field of Nanocrystal Nonvolatile Memories, are discussed.
\end{abstract}

Keywords- Quantum dots; Self-assembly; Nanopatterning; SiGe epitaxy; Vicinal surfaces; Focused Ion Beam; Nanocrystal nonvolatile memories.

\section{INTRODUCTION}

Epitaxially grown semiconductor quantum dots (QDs) deposited onto Silicon surfaces represent a nanotechnological area of focus for applications in nanomemories [1,2], nanoelectronics and optoelectronics [3]. Several approaches [4] have been tried so far to obtain various kinds of ordering of the dots. Groundbreaking routes for the controlled nanomanufacturing of dots (i.e. parallel fabrication with control over position, size, shape, and orientation at the nanometer scale) are more likely to result from bottom-up, self assembly processes resulting from natural phenomena, like the stress field occurring at $\mathrm{Si}-\mathrm{Ge}$ interface [5], rather than from customary lithographic techniques. The clever combination of self assembly with high resolution top-down patterning techniques, like Focused Ion Beam (FIB) Lithography [6] and Nanoindentation, facilitates the creation of technologically useful patterns of dots. In this perspective we have undertaken a study of feasible and relatively simple protocols to obtain ordered arrays of Ge QDs [7-8] on Si surfaces, to assess the growth kinetics of the dots and to quantify the attained ordering with computer-aided statistical analysis. Different techniques have been developed in other works to achieve long-range ordering of islands with a very narrow distribution of sizes in the case of $\mathrm{Ge} / \mathrm{Si}(001)$ system, such as growth of stacked multilayers of heteroepitaxial islands [9] or pulsed laser deposition nanostenciling [10], but the combination of self assembly and surface pre-patterning keeps showing compelling evidences of its high technological potential, particularly for its intrinsic high resolution. The idea here is to show the upsides and downsides (mainly from a nanotechnological point of view) of this approach through a discussion of every method we used to order Ge QDs on Si (Sections A and B). In Section $\mathrm{C}$ we present a technique used to evaluate the dots ordering on the surface which could easily be extended to the study of other systems and different nanostructures. A discussion of the state of the art in Ge Nanocrystal Nonvolatile Memories is presented in Section D.

\section{EXPERIMENTAL}

We hereby summarize the methods used to induce the nanopatterning of Ge QDs, classifying them as either "natural" methods (Section A), arising from naturally occurring surface structure and instabilities, or "artificial" methods (Section B) when a separate technique aided the ordering of the dots.

\section{A) Natural Methods \\ 1) Step bunching on $\mathrm{Si}(111)$}

On Si(111) surfaces, direct current heating may create bunches of natural surface steps depending on the temperature and on the mass transport regime [11], yielding a simple way to obtain a template for nanopatterned growth of Ge onto $\mathrm{Si}$ [12]. The step bunched (SB) clean surface consists of flat terraces separated by groups of monoatomic steps (Fig. 1a) acting as favoured nucleation sites due to local stress relaxation [13]. Ge was delivered on the SB surface via PVD technique and the samples were imaged in situ using Scanning Tunnel Microscopy (STM) in UHV. We analyzed the distribution and evolution of 3D Ge islands during the deposition process, with the substrate held at $450^{\circ} \mathrm{C}$. After the Wetting Layer (WL) formation, triangular islands nucleate and evolve at step edges rounding their corners up to complete ripening, thus merging in 1D structures. Subsequently, island nucleation takes place at the center of flat terraces, defining one-dimensional (1D) areas crowded with islands, in the form of ribbons running parallel to the step edges. This kind of growth causes 1D ordering (i.e. constant spacing) of the island-dense ribbons in the direction orthogonal to them, similarly to what a selective growth on a lithographically patterned surface would cause. The physical reason for this phenomenon lies in the capture of adatoms near 
step bunches, due to the high density of early nucleated dots, which impedes further nucleation in approximately 1D regions next to the steps.
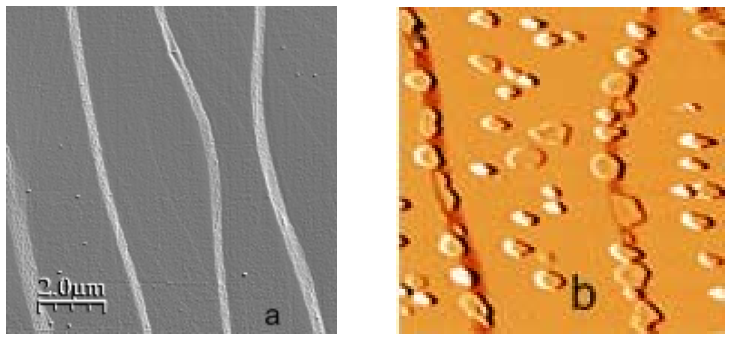

Figure 1. a) A clean, SB Si(111) surface imaged with STM. b) Regularly spaced ribbons with a high density of islands. The capture area defined from islands grown at step bunches prevents nucleation events nearby.

In the STM images Ge islands appear to be regularly spaced and fairly well aligned along the step edges, with an average distance depending on Ge coverage and terraces width. By varying the terrace width of the clean surface the number of rows of islands nucleated on the flat terraces can be controlled, and no growth is observed when the terrace width is lower than $\mathrm{w}_{\mathrm{dep}} \approx 1 \mu \mathrm{m}$ due to adatoms attraction towards step borders. Unfortunately, the islands average size $(100 \mathrm{~nm})$ still exceeds one of technological interest.

\section{2) Vicinal surfaces of Si (100)}

Vicinal surfaces of Si (100) present a stepped morphology allowing for the accommodation of the miscut angle.

The growth of strained structures such as Ge QDs usually occurs via the formation of a series of instabilities at the surface. In the case of Ge growth on low miscut $\mathrm{Si}(100)$ surfaces, different mechanisms have been proposed as responsible for such instabilities, like strain-induced step bunching [13], step-edge barriers [14] or diffusion anisotropy. At high miscut angles a complex situation is found where the interplay between incorporation of adatoms, surface reconstruction and anisotropic diffusion results in a periodic surface corrugation, that is, a rippled morphology. We studied different samples with a variety of miscut angles in the range $2^{\circ}-10^{\circ}$, whose systematic analysis is still underway. For example, in a recent paper [15] we discussed the formation of exquisitely aligned 1D SiGe structures, as reported in Fig. 2. These nanowires form as ripples on a vicinal $\mathrm{Si}(100)$ surface with $8^{\circ}$ miscut angle in the [110] direction. The clean surface is made up of $2.0 \pm 0.2 \mathrm{~nm}$ wide terraces bounded by $D_{B}$ steps.

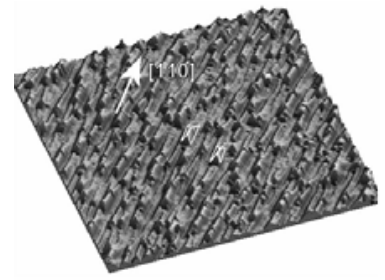

Figure 2. $500 \times 500 \mathrm{~nm}$ STM image of $3 \mathrm{D}$ view of corrugated surface showing aligned $1 \mathrm{D}$ structures.
After thermal annealing at $1200^{\circ} \mathrm{C}$, Ge was deposited by PVD at a growth temperature of $500^{\circ} \mathrm{C}$ with a constant flux of $1.25 \cdot 10^{-2} \mathrm{ML} / \mathrm{s}$ to suppress kinetic step bunching [16]. Threedimensional ripples bordered by two $\{105\}$ reconstructed facets (Fig. 3) form at 4ML coverage parallel to the [110] direction, mainly evolving from hut clusters through a step flow process in the miscut direction. At larger Ge depositions the ripples grow higher and longer, with an appealing 1D, well ordered appearance.

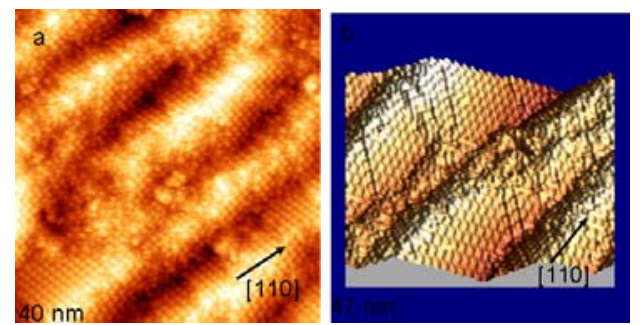

Figure 3. a) $40 \times 40 \mathrm{~nm}$ STM image showing ripples on an $8^{\circ}$ off Si (100) vicinal surface after $15 \mathrm{ML}$ Ge deposition. b) $50 \times 50 \mathrm{~nm}$ STM 3D image of $\{105\}$ reconstructed facets making up the nanowires.

Using samples with different miscut angle nanowires length and physical properties could in principle be tailored, but a final mastery of these aspects is not yet achieved. Si(111) also shows vicinal surfaces with remarkable instabilities [16,17], which so far have been poorly experimentally investigated and could provide new insights for this work.

\section{3) Ripples on $\mathrm{GeSi} / \mathrm{Si}(100)$ and role of surfactants}

Ripples similar to those described above can be created on highly misoriented by Ge-Si alloy deposition by MBE, and the wavelength of the undulations can be controlled by the $\mathrm{Si} / \mathrm{Ge}$ deposition flux ratio. These periodic ripples provide preferential nucleation centers for Ge QDs, which grow macroscopically aligned in the [110] direction when Ge is deposited on the corrugated surface [18]. The islands size can be diminished down to $50 \mathrm{~nm}$ with the aid of a solid surfactant like $\mathrm{Sb}$. In a typical procedure used to prepare these samples 10-15ML of $\mathrm{Ge}_{0.5} \mathrm{Si}_{0.5}$ are deposited via $\mathrm{MBE}$ at $600^{\circ} \mathrm{C}$ on a $10^{\circ}$ misoriented $\mathrm{Si}(100)$ vicinal surface. A template of periodic ripples with an average wavelength of $90 \mathrm{~nm}$ is thus obtained, and after a deposition of $0.5 \mathrm{ML}$ of $\mathrm{Sb}$ at $400^{\circ} \mathrm{C} \mathrm{Ge}$ is deposited to form chains of ultrasmall Ge islands (Fig. 4). These results demonstrate the capability of self-assembly strategies for 1D alignment of QDs [19] and confirm small quantities of surfactants can dramatically alter nanostructures morphology.

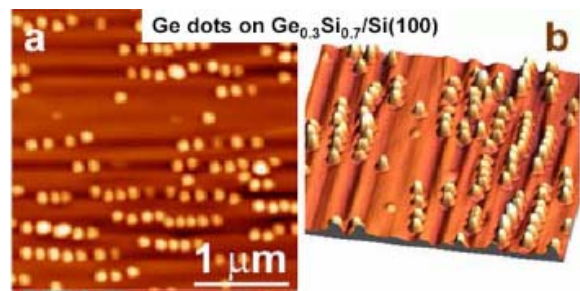

Figure 4. Remarkably aligned QDs grown on a SiGe overlayer deposited onto a $10^{\circ}$ off $\mathrm{Si}(100)$ vicinal surface. The use of $\mathrm{Sb}$ halves the size of the islands shown here [18]. 


\section{B) Artificial methods}

\section{1) STM nanopatterning via tip nanoindentation}

We produced arrays of pits in $\operatorname{Si}(100)$ surface at $500^{\circ} \mathrm{C}$ by suddenly approaching the surface with the STM tip at selected locations, while the z-feedback was switched off. This patterning technique allows for very high resolution, almost contamination free patterning, but is extremely timeconsuming. The so produced patterns consist of several holes with diameter in the range $8-15 \mathrm{~nm}$ and depths of $1-2 \mathrm{ML}$, with a mutual distance of $60 \pm 5 \mathrm{~nm}$ (Fig. 5). The holes serve as preferred nucleation sites for islands growth due to local stress relieving by step-step interaction. We followed in real-time the WL growth observing a 2D-3D strain induced transition at 3-4 ML Ge coverage, while the WL does not penetrate inside the pores. Two different stages of islands growth were identified: the first stage corresponds to a pre-pyramid and the second one to a pyramidal hut cluster. Quantitative information on the growth mechanism can be obtained from the time evolution of the dots volume [20].

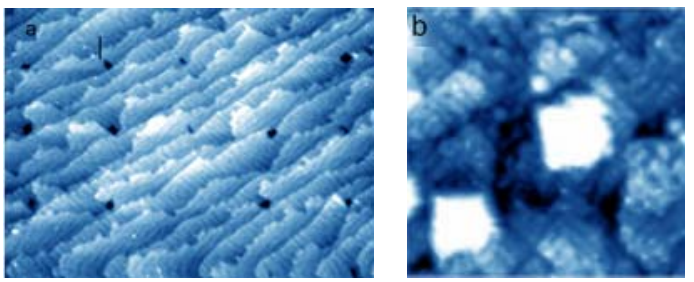

Figure 5. a) STM image of an array of pits produced by STM nanolithography. b) Selective nucleation of Ge clusters by a pit.

\section{2) Focused Ion Beam patterning}

We studied the growth process of Ge QDs both on bare and oxidized $\mathrm{Si}(100)$ substrates patterned with FIB [21]. Several patterns of holes with square symmetry were produced by FEI Company on bare $\mathrm{Si}(100)$ substrate with squares side of $780 \pm 30 \mathrm{~nm}$ and $500 \pm 30 \mathrm{~nm}$ and a total area of several $\mu \mathrm{m}^{2}$. A well established protocol for samples cleaning was used to reduce the contamination of $\mathrm{Ga}$ ions below $10^{16} \mathrm{~cm}^{-3}$. We then collected STM images during the growth, separately on patterned and nonpatterned areas, and compared QDs ordering in different substrate regions. On prepatterned regions the holes provide preferred nucleation centers for the islands, thus facilitating the creation of ordered arrays. A first population of Ge islands nucleates at pits edges and later covers the whole hole. Only at a later growth stage do the islands nucleate between previously formed dots, thus determining a bimodal distribution in dots size [21]. A striking difference in the ordering between patterned and nonpatterned areas was found. Similar experiments were carried out on oxidized $\mathrm{Si}(100)$ with $20 \mathrm{~nm}$ of tunnel thermal oxide. The surface was patterned with a dense (up to $5 \cdot 10^{10} \mathrm{~cm}^{-2}$ ) array of holes with FIB, with a pitch distance of $48 \pm 3 \mathrm{~nm}$ and holes diameter of $30 \pm 2 \mathrm{~nm}$ as measured ex situ by AFM. We deposited an amorphous layer of Ge by PVD at room temperature; after thermal annealing at $600^{\circ} \mathrm{C}$ for $1 \mathrm{~h}$ small $\mathrm{Ge}$ droplets are formed, nucleating at the patterned holes.
QDs densities as large as $4.3 \cdot 10^{10} \mathrm{~cm}^{-2}$ were measured by AFM after the completed deposition-annealing cycle. The same experiment was repeated on an oxidized, nonpatterned surface allowing a quantitative comparison of islands ordering, as discussed in the following section.

\section{C) Statistical Analysis : Pair Distribution Function}

We performed a quantitative comparison of QDs ordering on two samples prepared with the method explained above. Two resulting $2 \times 2 \mu \mathrm{m}$ images (Fig. 6) were compared, one of them corresponding to Ge dots formed on a patterned area and the other one to a pattern-free sample; both were taken after $\mathrm{Si}(100)$ oxidation and deposition-annealing cycle.

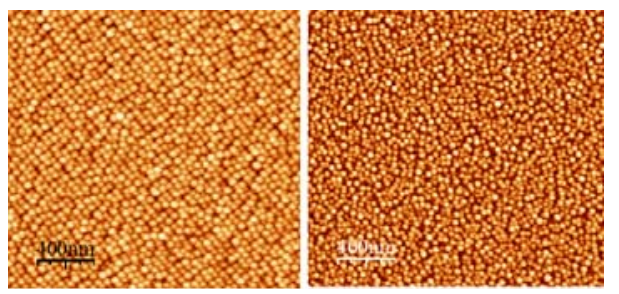

Figure 6. Ge islands on oxidized $\mathrm{Si}(100)$ surface. Left: FIB patterned sample, showing arrangement of QDs with a square symmetry similar to that of the underlying holes pattern. Right: nonpatterned sample, with average dots sizes of $35 \mathrm{~nm}$ and average distance between neighbour dots of 50nm [21].

Although it is apparent from Fig. 6 that the patterned sample shows superior order, we want to establish the nature of this order by assessing the 2D Pair Distribution Function (PDF) [22], which for our computational scopes we define as:

$$
g(r)=\frac{N(r)}{2 \pi r \Delta r \rho}
$$

where $N(r)$ is the number of dots found in the circular crown at distance $r$ of area $2 \pi r \Delta r$, and $\rho$ is the mean dots density in the image. The PDF as defined in (1) has an asymptotic value of one, and deviations from unity have to be interpreted as fluctuations from the mean density at that particular distance from a dot, within a bin size $\Delta r$. We calculate the PDF of the two distinct dots distributions in Fig. 6 using a software routine developed by Del Moro et al. [23] which correctly accounts for edge effects. The $g(r)$ function is obtained from an histogram of the distances found between the centers of mass of dots pairs. With reference to Fig. 7, we observe that for the nonpatterned sample a single peak is found at the nearest neighbour distance of approx. $50 \mathrm{~nm}$, whereas the patterned sample shows several peaks due the long range order of the dots, each peak corresponding to a single or multiple (depending on the chosen bin size) nearest neighbour shell of dots. This result shows that a long range order is attained in the patterned sample. We speculate that in the nonpatterned sample the dots pair potential rules the minimum interdot distance, showing an effect of short range self-ordering extending only to the first nearest neighbour shell; when a holes pattern is present, the pattern itself rather determines the nucleation sites and therefore the interdot distance. 

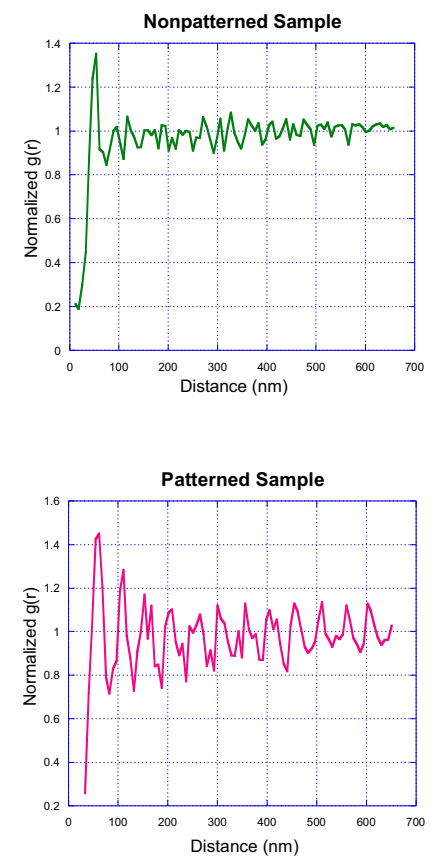

Figure 7. PDF of nonpatterned (top) and patterned (bottom) samples. Respectively, short-range order and long-range order is found.

\section{D) Ge Nanocrystal Nonvolatile Memories}

Current float-gate design of nanomemories limits the scaling of the device to the $65-\mathrm{nm}$ node since a relatively thick oxide is needed for charge retention. Si nanocrystal non-volatile memories promise to enable further scaling of the tunnel oxide without sacrificing charge retention [1]. It has been calculated that Ge QDs based nanocrystal memories should be superior to $\mathrm{Si}$ based devices [2], allowing longer retention time due to higher electrons confinement. Nonetheless, a minimum dots density of $10^{11}-10^{12} \mathrm{~cm}^{-2}$ is needed, and for good device performance the dots should be laterally isolated and have uniform size and areal density. Our results show promising routes for direct Ge dots deposition; although a 10-fold QDs density increase would be needed, lateral islands isolation and constant areal density are obtained with FIB patterning. Test devices were prepared by depositing a thick control oxide and a metal gate on QDs patterns, and their characterization showed encouraging results.

\section{CONCLUSION}

We have presented several routes for the self-assembly of technologically interesting zero-dimensional and onedimensional SiGe nanostructures. A combination of StranskyKrastanov growth of $\mathrm{Ge}$ on $\mathrm{Si}$ and naturally occurring surface instabilities proved essential. Ordering in 1D and 2D of Ge QDs is also shown, the latter being achieved with the aid of top-down techniques such as FIB and STM lithography. A quantitative evaluation of islands ordering showed the existence of short-range order on nonpatterned samples and of long range order on FIB patterned substrates. This stimulates us to produce denser FIB patterns to obtain dots sizes and densities of technological interest.

\section{REFERENCES}

[1] J. De Blauwe, IEEE Transactions on Nanotechnology, Vol. 1, No. 1, 2002, pp. 72-77.

[2] Y. Zhu, D. Zhao, J. Liu, J. Appl. Phys., Vol. 101, 2007, 034508.

[3] Y. Kuo, Y.K. Lee, Y. Ge, S. Ren, J.E. Roth, T.I. Kamins, D. Miller, J.S. Harris, Nature, 437, 2005, pp. 1334-1336.

[4] N. Motta, A. Sgarlata, A. Balzarotti, P.D. Szkutnik, Proc. of SPIE Vol. 6036, 2006, 603601-1.

[5] B. Voigtlander, Surf. Sci. Rep. 43, 2001, 127-254.

[6] A. Portavoce, R. Hull, M.C. Reuter, F.M. Ross, Phys. Rev. B, Vol. 76, 2007, 235301.

[7] N. Motta, P.D. Szkutnik, M. Tomellini, A. Sgarlata, M. Fanfoni, F. Patella, A. Balzarotti, C.R. Physique, Vol. 7, 2006, pp. 1046-1072.

[8] F. Patella, A. Sgarlata, F. Arciprete, S. Nufris, P. Skutznik, E. Placidi, M. Fanfoni, N. Motta, A. Balzarotti, J. Phys. Cond. Matt., Vol 16, 2004, S1503-S1534.

[9] G. Capellini, M. De Seta, C. Spinella, F. Evangelisti Appl. Phys. Lett., Vol. 82, 2003, p. 1772.

[10] C.V. Cojocaru, A. Bernardi, J. S. Reparaz, M. I. Alonso, J. M MacLeod, C. Harnagea, F. Rosei, App. Phys. Lett., Vol. 91, 2007, 113112.

[11] J.J. Metois, S. Stoyanov, Surf. Sci. 440, 1999, 407-419.

[12] A. Sgarlata, P. D. Szkutnik , A. Balzarotti, N. Motta, F.Rosei, Appl. Phys. Lett., Vol. 83, 2003, p. 4002.

[13] J. Tersoff, Y.H. Phang, Z. Zhang, M.G. Lagally, Phys. Rev. Lett., Vol. 75, 1995, p. 2730.

[14] A. Pimpinelli, A. Videocoq, Surf. Sci. 445, 2000, L23.

[15] P.D. Szkutnik, A. Sgarlata, A. Balzarotti, N. Motta, A. Ronda, I. Berbezier, Phys. Rev. B, Vol. 75, 2007, 033305.

[16] J. Mislivecek, C. Schelling, F. Schäffler, G. Springholz, P. Smilauer, J. Krug, B. Voigtlander, Surf. Sci. 520, 2002, p. 193.

[17]H.-C. Jeong, E.D. Williams, Surf. Sci. Rep. 34, 1999, pp. 171-294.

[18] I. Berbezier, A. Ronda, A. Portavoce, N. Motta, Appl. Phys. Lett., Vol. 83, 2003, p. 4833.

[19] I. Berbezier, A. Ronda, Phys. Rev. B, Vol. 75, 2007, 195407.

[20] P.D. Szkutnik, A. Sgarlata, S. Nufris, N. Motta, A. Balzarotti, Phys. Rev. B, Vol. 69, 2004, 201309(R).

[21] A. Sgarlata, A. Balzarotti, I. Berbezier, P. Szkutnik, F. Rosei, N. Motta, Proc. 2006 International Conference on Nanoscience and Nanotechnology (ICONN 2006), p.328 (on CD) IEEE Cat. No. 06EX1411C.

[22] J.P. Hansen, I.R. McDonald, Theory of Simple Liquids, 2nd ed., Academic Press, 1990.

[23] F. Berrilli, D. Del Moro, G. Consolini, E. Pietropaolo, T.L. Duvall, Jr., A.G. Kosovichev, Solar Physics, vol. 221, Issue 1, 2004, pp. 33-45. 